\title{
Descubriendo el lenguaje a través de la realidad aumentada y la pizarra digital'
}

\author{
Discovering Language through Augmented Reality and the Interactive Digital White Board ${ }^{2}$ \\ Descobrir a linguagem através da realidade aumentada e lousa digital ${ }^{3}$
}

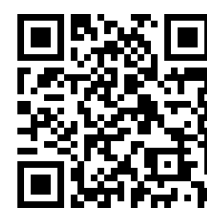

\author{
Sandra Ruperta Pérez-Lisboa 4 \\ Universidad de Playa Ancha \\ San Felipe, Chile \\ sandra.perez@upla.cl \\ http://orcid.org.0000-0002-0389-7242
}

Recibido 27 de abril de 2016 • Corregido 1 de agosto de 2017 • Aceptado 25 de agosto de 2017

Received 27 de abril de 2016 • Revised 1 de agosto de 2017 • Accepted 25 de agosto de 2017

Recebido 27 de abril de 2016 • Revisado 1 de agosto de 2017 • Aprovado 25 de agosto de 2017

\begin{abstract}
Resumen: El presente estudio analizó el desarrollo de los aspectos fonológico, semántico y sintáctico utilizando la realidad aumentada y la pizarra digital interactiva, en niños y niñas del Nivel Transición Mayor del Liceo San Felipe. Con la implementación de estas herramientas se llevaron a cabo experiencias de aprendizajes con el alumnado de párvulos, potenciando la comprensión de oraciones y palabras en sus componentes sucesivos, la segmentación lingüística, conciencia fonológica y la reflexión sobre el significado de las palabras y oraciones. Las experiencias se realizaron en el aula didáctica de la carrera de Educación Parvularia de la Universidad de Playa Ancha, campus San Felipe,
\end{abstract}

\footnotetext{
${ }^{1}$ Esta investigación se realizó con la colaboración del docente Javier Castillo Allaria, Ingeniero Informático y las estudiantes Karon Arancibia Orrego y Paulina Cáceres Méndez.

${ }^{2}$ his research was carried out with the collaboration of the teacher Javier Castillo Allaria, Computer Engineer, and the students Karon Arancibia Orrego and Paulina Cáceres Méndez.

${ }^{3}$ Esta investigação foi realizada com a colaboração do professor Javier Castillo Allaria, engenheiro de informática e de estudantes Karon Arancibia Orrego e Paulina Cáceres Méndez.

${ }^{4}$ Magíster en Educación, mención en Pedagogía y Gestión Universitaria de la Universidad Metropolitana de Ciencias de la Educación. Educadora de Párvulos de la Pontificia Universidad Católica de Chile. Psicopedagoga de la Universidad Mayor. Diplomado en Pedagogía Universitaria y Diplomado en Gestión de la Universidad Metropolitana de Ciencias de la Educación. Especialista en el Programa de Enriquecimiento Instrumental de Dr. Feuerstein - Primer nivel. Docente del Departamento de Educación y Pedagogía de la universidad de Playa Ancha, campus San Felipe. Actualmente realiza proyecto de investigación "Realidad aumentada y pizarra digital interactiva en la construcción de habilidades científicas. Una propuesta metodológica para niños y niñas del nivel transición mayor en el aprendizaje de la física". Se ha presentado en congresos nacionales e internacionales en el área del desarrollo del lenguaje, la enseñanza de las ciencias naturales en educación parvularia y las tecnologías.
} 
doi: http://dx.doi.org/10.15359/ree.21-3.14

URL: http://www.una.ac.cr/educare

CORREO: educare@una.cr

con una duración de 60 minutos una vez a la semana por cuatro meses. El tipo de estudio fue cuasi experimental y a través de un pre y postest, se comprobó el avance que tuvieron 18 estudiantes de un liceo municipal de la comuna de San Felipe. Los instrumentos utilizados fueron la prueba de segmentación lingüística, prueba de exploración del lenguaje comprensivo y expresivo (ELCE); subtest aspecto semántico, prueba evalúa O; subtest palabras y frases. Los resultados obtenidos, basados en la comparación de pre y postprueba, muestran cambios en el manejo de los aspectos semánticos, sintácticos y fonológicos logrados por los niños y las niñas con esta metodología. Sin embargo, se requiere más investigación para validar esta propuesta en la enseñanza de la metalingüística.

Palabras claves: Aula didáctica; conciencia fonológica; conciencia semántica; conciencia sintáctica y herramientas tecnológicas.

Abstract: This study analyzed the development of phonological, semantic, and syntactic aspects by using augmented reality and interactive whiteboard with boys and girls in the kindergarten of Liceo San Felipe, San Felipe, Chili. With the implementation of these tools, learning experiences were carried out, enhancing the understanding of sentences and words in their successive components: linguistic segmentation, phonological awareness, and reflection on the meaning of words and sentences. The experiments were carried out in a didactic classroom of the course of Educacion Parvularia (PreSchool Education) at the University of Playa Ancha, San Felipe Campus, for 60 minutes, once a week for four months. It was a quasi-experimental study, and through pre- and post-tests, it was possible to verify the development of 18 children of a municipal school in San Felipe. The instruments used were the Linguistic Segmentation Test, Comprehensive and Expressive Language Examination Test (ELCE); Subtest semantic aspect, Test Evaluation O; Subtest words and phrases. The results, based on the comparison of pre- and post-test, showed changes in the management of the semantic, syntactic, and phonological aspects achieved by the children with this methodology. However, more research is needed to validate this proposal in teaching metalinguistic.

Keywords: Classroom teaching; phonological awareness; semantic awareness; syntactic awareness and technological tools.

Resumo: Este estudo analisou o desenvolvimento dos aspectos fonológico, semântico e sintático através da utilização de realidade aumentada e quadro interactivo em crianças no jardim da infância do Colégio San Felipe. Com a utilização dessas ferramentas, foram realizadas experiências de aprendizagem com os alunos do jardim de infância, melhorando a compreensão de frases e palavras em seus componentes sucessivos: segmentação linguística, consciência fonológica e reflexão sobre o significado das palavras e frases. Os experimentos foram realizados em sala de aula como parte do curso de Educação Infantil da Universidade de Playa Ancha, Campus San Felipe, com duração de 60 minutos, uma vez por semana durante quatro meses. $O$ tipo de estudo foi quase experimental e através de pré e pós-testes comprovou-se o progresso de 18 crianças do Colégio San Felipe. Os instrumentos utilizados foram Teste de Segmentação Linguística, Teste de Exploração e Compreensão da Linguagem Expressiva (ELCE); sub teste aspecto semântico, avaliação de teste O; sub teste palavras e frases. Os resultados, com base na comparação dos pré e pós-testes, mostraram mudanças nos aspectos semântico, sintático e fonológico obtidas pelas crianças por meio desta metodologia. No entanto, são necessárias mais pesquisas para validar esta proposta no ensino de metalinguagem

Palavras-chave: Ensino na sala de aula; consciência fonêmica; consciência semântica; consciência sintática e ferramentas tecnológicas. 


\section{Introducción}

La educación parvularia en Chile, integra desde el año 2003 el uso de las tecnologías de la información y comunicación (TIC) a las experiencias de aprendizajes como un recurso de apoyo. Estas herramientas se ponen al servicio del trabajo pedagógico al ofrecer a la niñez interrelaciones que son parte de los procesos de comunicación de cada niño y niña y entre sus pares (Enlaces Mineduc, 2016).

Una serie de estudios (Alegría, 1985, 2006; Bravo, 1990) confirman que la conciencia fonológica es el predictor más fuerte de los avances en las etapas iniciales de los aprendizajes de la lectura y la escritura. Por ello, Orellana (2000) señala que se deben potenciar los aspectos metalingüísticos en los niños y niñas; del acceso al contenido, aspectos semánticos, los que tienen relación con la estructura, aspectos sintácticos y los concernientes con la organización alfabética de la lengua, los aspectos fonológicos.

El propósito de este estudio fue favorecer en los niños y niñas el desarrollo de los aspectos fonológico, semántico y sintáctico con herramientas TIC, en el aula didáctica de la carrera de Educación Parvularia de la Universidad de Playa Ancha, Campus San Felipe. Esta investigación se orienta con las siguientes preguntas: ¿cómo se desarrolla la habilidad para identificar, segmentary articular fonemas?, ¿de qué manera se potencia la comprensión de oraciones y palabras en sus componentes sucesivos?, ¿cómo se desarrolla la reflexión sobre el significado de las palabras y oraciones?

\section{Revisión bibliografía}

Actualmente las tecnologías de la información y la comunicación se relacionan de forma más directa con las personas, ya que estas se vinculan en ambientes digitalizados. Todas las empresas de servicios públicos o privados se sustentan en el uso y soporte de plataformas tecnológicas, las que facilitan la eficiencia y productividad del país, tanto en lo social como en lo económico.

Asimismo, los niños y las niñas de hoy nacen con una facilidad para acceder y manejar diferentes herramientas digitales, ya en el vientre materno se ven invadidos por la tecnología a través de exámenes médicos, imágenes y sonidos, para luego tener interacción con celulares, computadores, televisores, discos compactos, electrodomésticos u otros, lo cual permitirá poseer un pensamiento más avanzado (Garassini y Padrón, 2004), son capaces de hacer varias tareas al mismo tiempo y en periodos más cortos, ya que están creciendo en un ambiente con una fuerte estimulación visual y auditiva.

Hace unas décadas atrás, se comenzó a implementar la informática en educación para generar nuevas propuestas educativas, con el objetivo de provocar cambios didácticos dentro del aula. Según el informe de la Unesco (2016), su uso ha permitido a las organizaciones educativas mejorar resultados cuando los programas tienen claros propósitos educativos, preparando adecuadamente al profesorado para que rompa los viejos moldes y cree nuevas formas de trabajo y funcionamiento. 
doi: http://dx.doi.org/10.15359/ree.21-3.14

URL: http://www.una.ac.cr/educare

CORREO: educare@una.cr

\section{Las TIC en educación parvularia}

La educación parvularia está constantemente realizando programas de mejoramiento de su currículo por la relevancia que tienen los aprendizajes en los primeros años de vida. De tal forma, y para mejorar las prácticas pedagógicas, las bases curriculares integran en el trabajo pedagógico el uso de las tecnologías de la información y comunicación como un recurso de apoyo al aprendizaje. En los últimos años se han integrado los netbook y las pizarras digitales al aula, a partir del 2016 comienzan a trabajar con tablet para potenciar las relaciones lógicas matemáticas y la cuantificación (Enlaces Mineduc, 2016).

Los estudios de Hirumi \& Grau (1996) dan a conocer que la utilización de las TIC con animaciones y movimientos pueden representar mejor algunos acontecimientos, de ahí la relevancia de incorporar estos a la experiencia pedagógica en la educación parvularia. Es así como el uso de las TIC se debe integrar al trabajo pedagógico, como otra estrategia metodológica. Por ello la red de Enlaces (2016) está realizando distintos programas en educación preescolar. Estos desafíos siguen abriendo un amplio espectro de divulgación y aplicación de esta política, lo que implica que cada establecimiento educacional tenga soporte, mantención y capacitación para personal docente y técnico en párvulos.

\section{La realidad aumentada}

La realidad aumentada (RA) se define como "visión directa o indirecta de un entorno físico del mundo real, cuyos elementos se combinan con elementos virtuales para la creación de una realidad mixta a tiempo real" (Yanac 2011, p. 3, citando a Villarreal y Heras, 2007). Esta herramienta tecnológica usa objetos en tres dimensiones (3D) organizados por el computador sobre imágenes reales en tiempo real.

Para Azuma (1997), los sistemas de realidad aumentada son aquellos que poseen: combinación entre realidad y virtualidad; tridimensionalidad; interacción en tiempo real. Por ello que esta tecnología está presente en diferentes dispositivos móviles que permiten jugar, obtener información, guías de museo y marketing. Así también en el último tiempo se han incorporado a la educación, de manera trepidante por el fácil acceso de estas aplicaciones.

Diversos estudios avalan la aplicación de esta tecnología basada en realidad aumentada, como lo realizado por Kerawalla, Luckin, Selijefot y Woolard (2006), quienes señalan que a través de la RA el estudiantado se compromete, se motiva y explora los materiales en clases. Asimismo, según lo investigado por Shelton y Hedley (2002), el estudiantado logró comprender los contenidos espaciales complejos, a través de información visual y sensorial. Como también los estudio de Klopfer y Squire (2008), Billinghurst (2002), Cooperstock (2001) desarrollaron diferentes entornos de aprendizajes con esta herramienta. 


\section{La pizarra digital interactiva}

La pizarra digital interactiva se puede definir como "una pantalla táctil que permite interactuar y hacer anotaciones sobre ella con un puntero y a veces incluso con los dedos" (Noda, 2009, p. 122), lo cual se transforma en una herramienta tecnológica potente para la construcción de nuevos aprendizajes.

Variadas investigaciones reconocen la importancia de la pizarra, una de ellas es la de Andrade (2012), al analizar esta herramienta en la enseñanza de competencias en el área de las matemáticas. Los resultados indicaron que este recurso se fue haciendo necesario para docentes, porque las clases eran más motivadoras, y para estudiantes era de gran interés utilizarla por el desenvolvimiento que les permite en las clases.

Por otro lado, la investigación de Marquès y Casals (2003) les permitió identificar los beneficios del uso de la pizarra tanto para personal educativo como para estudiantes, quienes señalan que con esta herramienta pueden observar y comentar, de manera interactiva, colectiva y dinámica, toda la información que les proporciona internet, televisión o cualquier otra tecnología. Ahora bien, Briede, Leal, Mora y Pleguezuelos (2015) señalan que "la pizarra digital interactiva (pdi) como plataforma de proyección pública, pantalla interactiva y registro a través del almacenamiento digital aporta a la sociabilización e inmediatez dialéctica, favoreciendo también la retroalimentación de los conceptos, croquis y proceso creativo" (p. 15).

Los resultados de estas y otras investigaciones evidencian que con el uso de esta herramienta se obtienen innumerables posibilidades de innovar en las prácticas pedagógicas, con el propósito de motivar al estudiantado en el proceso de aprender, teniendo en cuenta la diversidad, características y necesidades actuales de cada quien.

\section{La enseñanza de la metalingüística}

La metacognición se puede definir como "...la aplicación del pensamiento al acto de pensar" (Pinzás, 2003, p. 24), lo que implica la comprensión de los fenómenos cognitivos. Por consiguiente, los componentes de la metacognición, según Garton (1994), son la habilidad para entender y pensar de las propias experiencias cognitivas y tomar conciencia de las situaciones para invocarlas y desarrollarlas.

Ahora bien, la metalingüística representa la comprensión sobre la lengua (Medina, 1993), para ello se debe reflexionar sobre el lenguaje, separando la forma del significado. En este contexto, Orellana (2000) señala que se deben potenciar los aspectos en los niños y las niñas del acceso al contenido, aspectos semánticos, los que tienen relación con la estructura, aspectos sintácticos y los concernientes con la organización alfabética de la lengua, los aspectos fonológicos. 
doi: http://dx.doi.org/10.15359/ree.21-3.14

URL: http://www.una.ac.cr/educare

CORREO: educare@una.cr

El aspecto semántico "...consiste en el estudio de los significados de palabras y la forma en que ellas se relacionan entre sí en el léxico mental" (Orellana, 2000, p. 17). Se relaciona con la toma de conciencia y la organización de los contenidos lingüísticos en la memoria y con la fluidez para establecer relaciones significativas que tenga la persona.

Ahora bien, el aspecto fonológico es la toma de conciencia de las unidades mínimas que componen las emisiones lingüísticas de la lengua. La niñez no descubre, por sí misma, los fundamentos psicolingüísticos que llevaron a la invención del lenguaje, necesita un proceso sistemático para que reconozca la lengua (Bravo, Villalón y Orellana, 2002).

A su vez, el aspecto sintáctico es la toma de conciencia que facilita la organización de las ideas en expresiones claras y precisas. La ejercitación en la niñez le permite comprender las diferentes partes de la oración y la función que cumple cada una en el texto total (Díaz y Álvarez-Salamanca, 2006).

\section{Materiales y métodos}

Esta investigación es de tipo cuasi experimental, desde un enfoque cuantitativo, midiendo una intervención antes y después de la aparición de algún factor de tipo casual. En este contexto se enfoca a un diseño de pre prueba-post prueba con un solo grupo, al que se aplica una prueba previa al estímulo, después se realiza el tratamiento y finalmente se le aplica una prueba posterior a este (Hernández, Fernández y Baptista, 2014). En este caso, a través de las pruebas se analizará el nivel de la niñez en los aspectos fonológicos, semánticos y sintácticos. Para ello, se utilizaron los instrumentos estandarizados: Prueba de segmentación lingüística, prueba de exploración del lenguaje comprensivo y expresivo (ELCE); subtest aspecto semántico, prueba evalúa O; subtest palabras y frases.

En este estudio participó la niñez del liceo San Felipe ${ }^{5}$, la muestra se determinó a través del tipo no probabilística, la selección dependió de las características del estudio (Hernández, et al., 2014). En este contexto, se eligió a los 18 párvulos y párvulas del nivel Transición Mayor, los cuales fueron intervenidos en el aula didáctica de la carrera de Educación Parvularia de la Universidad de Playa Ancha, Campus San Felipe.

\section{Procedimiento}

El aula didáctica está equipada con las herramientas tecnológicas de realidad aumentada y pizarra digital. El sistema de realidad aumentada funciona con un PC, proyector y cámara, para exhibir los objetos de 3D por medio de marcadores predeterminados. La pizarra digital tiene un puntero que es empleado por los niños y niñas en las experiencias que se les presentan con diversas animaciones, vídeos, PowerPoint, páginas web y software educativo según el objetivo de aprendizaje.

${ }^{5}$ Los padres, madres y apoderados de los niños y niñas que participaron en esta investigación, dieron el consentimiento informado para su evaluación.

6 
Las sesiones se realizaron una vez por semana, con una duración de sesenta minutos, las intervenciones duraron cuatro meses aproximadamente. Para lograr el objetivo propuesto, se planificaron 15 experiencias de aprendizaje en torno a diferentes contenidos, coordinados con la educadora de párvulos del nivel, se trabajó con las vocales y las consonantes m, p, l, s, t y d. En cada experiencia de aprendizaje se potenciaban los tres aspectos: la segmentación lingüística y conciencia fonológica; la comprensión de oraciones y palabras en sus componentes sucesivos; la reflexión sobre el significado de las palabras y oraciones.

\section{Resultados}

A los dieciocho niños y niñas que fueron intervenidos en el aula, se les aplicaron tres instrumentos para comprobar el avance en los aspectos fonológicos, semánticos y sintácticos. A continuación, se da a conocer el análisis de estos.

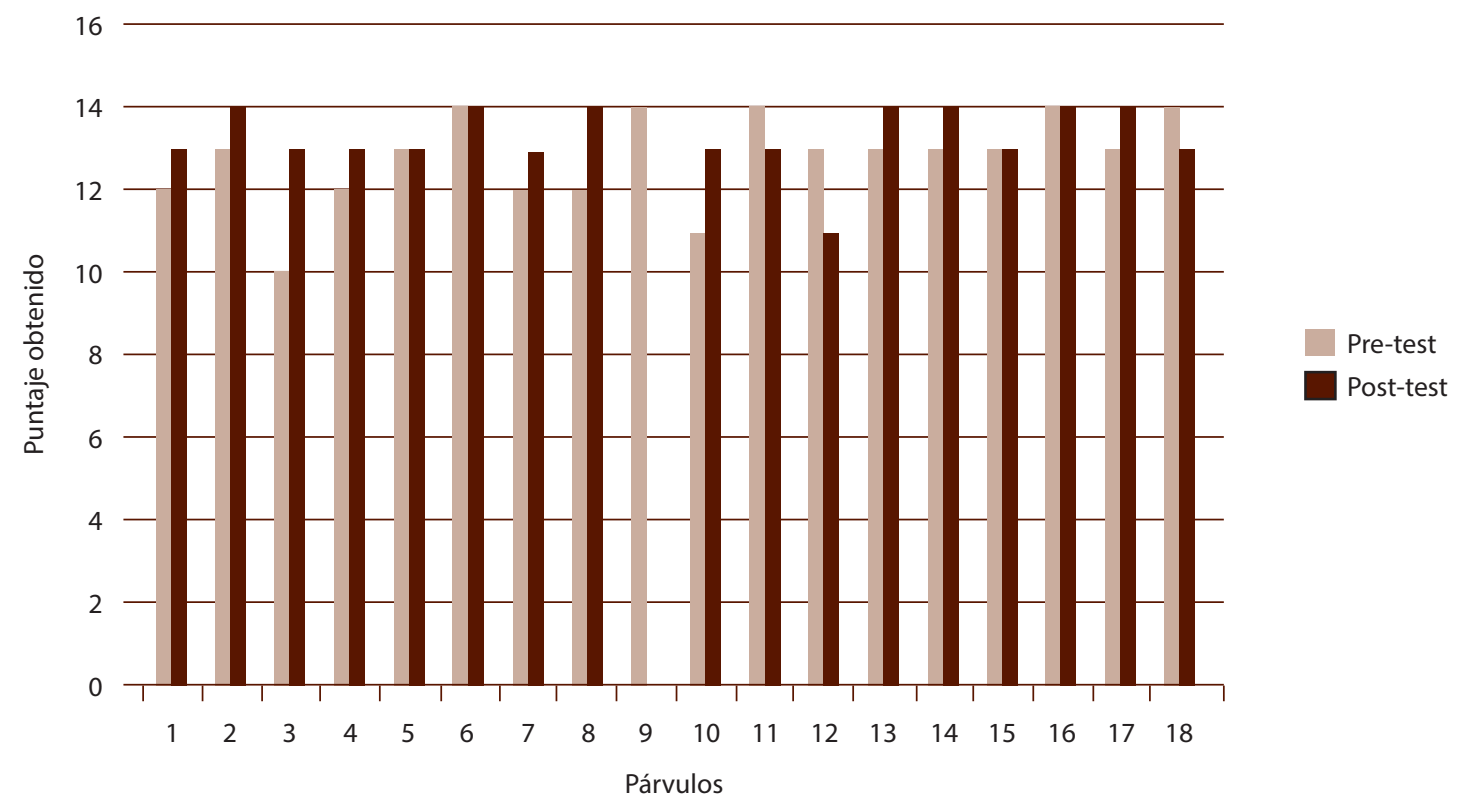

Figura 1: Resultados evaluación del aspecto fonológico.

En la aplicación de la prueba de segmentación lingüística, la Figura 1 da cuenta que la mayoría de la niñez mejoró sus resultados en comparación con el pre-test (cabe destacar que el niño 9 no pude ser evaluado en el post-test), esto implica que fueron tomando conciencia de que las palabras están formadas por fonemas y sílabas. Ahora bien, se evidencia, además, que hay párvulos y párvulas (4, $11,12,18)$ que disminuyeron el puntaje de la prueba, esto se debe a que tuvieron un alto porcentaje de inasistencia al aula didáctica, variable que no se consideró en la investigación. 
doi: http://dx.doi.org/10.15359/ree.21-3.14

URL: http://www.una.ac.cr/educare

CORREO: educare@una.cr

Este resultado permite reflexionar sobre el aporte de la realidad aumentada en el aspecto fonológico, según lo que señala Kerawalla, et al. (2006), con esta herramienta los niños y las niñas exploran los materiales presentados. Estos materiales como animales, personas y objetos que se iban relacionando con las vocales y consonantes, los párvulos y las párvulas iban tomando conciencia de las unidades mínimas que poseen las emisiones lingüísticas (Bravo et al., 2002). Asimismo las experiencias de aprendizajes presentadas en la pizarra digital fueron más motivadoras (Andrade, 2012) para este reconocimiento.

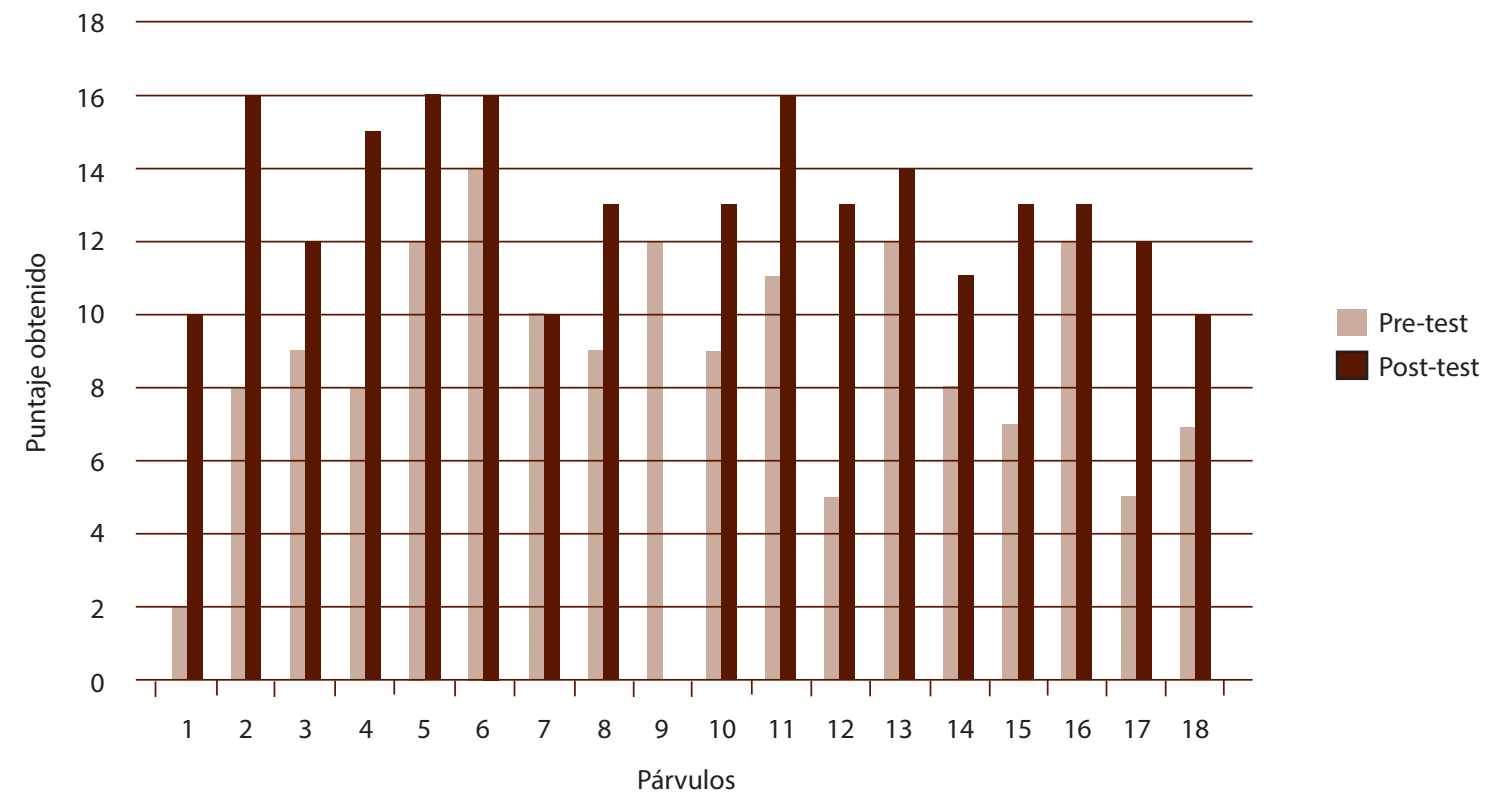

Figura 2: Resultados evaluación del aspecto semántico.

Ahora bien, en el aspecto semántico, la Figura 2 demuestra el gran avance que todos los niños y todas las niñas lograron con el trabajo pedagógico del aula, en el cual debieron proponer concepto a una categoría, descubrir el concepto que no correspondía de una categoría, completar analogías y asignar elemento a una categoría. Estas experiencias de aprendizajes permitieron a los párvulos y a las párvulas establecer redes semánticas para comprender los mensajes, conectándolos con los conocimientos previos que tienen en su memoria (Orellana, 2000). Según Marquès y Casals (2003), a través de la pizarra digital se puede observar, comentar de forma más interactiva, dinámica y colectiva la información recibida, lo cual influyó en que los niños y niñas fueran comprendiendo el significado, sentido e interpretación de palabras y expresiones o símbolos. 


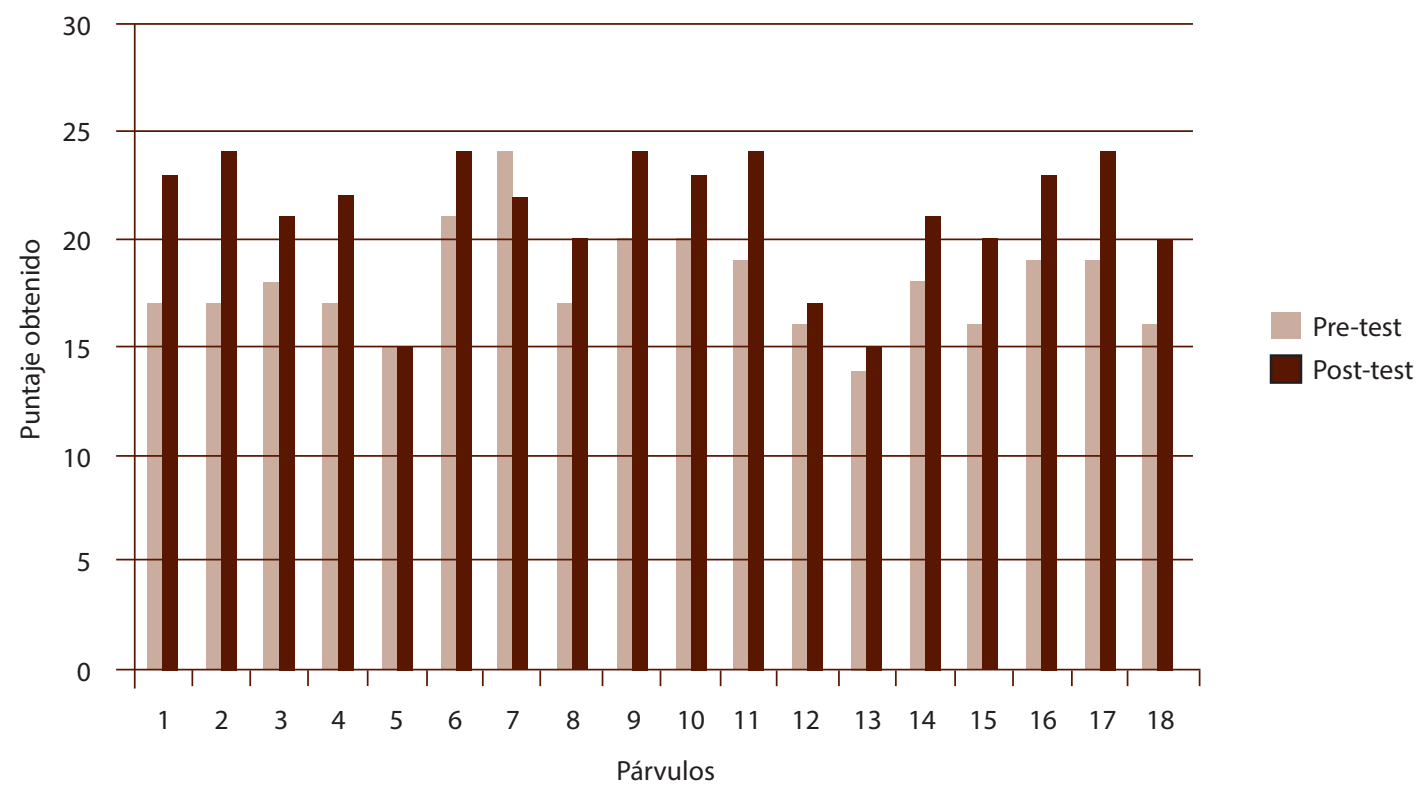

Figura 3: Resultados evaluación del aspecto sintáctico.

En relación con el aspecto sintáctico, la Figura 3 da a conocer que todos los niños y todas las niñas lograron un avance en esta área. El trabajar de forma sistemática la organización de los contenidos lingüísticos permitió a los párvulos y las párvulas entregar mensajes consecuentes con su interés, para Shelton y Hedley (2002), la realidad aumentada entrega información visual y sensorial que estimula el interés en los aprendizajes.

Por otro lado, con la pizarra digital se pudieron retroalimentar los conceptos en las presentaciones de vídeos, páginas web, animaciones, PowerPoint y software educativo, para luego crear diferentes oraciones que permitieron comprender sus diferentes partes (Díaz y Álvarez-Salamanca, 2006).

Finalizando el análisis de los resultados, con base en los puntajes obtenidos en el pos-test al contrastarlo con el pre-test, se logra inferir que hubo un gran avance en el desarrollo de los aspectos fonológicos, semánticos y sintácticos en las niñas y los niños que se intervinieron.

Como se observa en la Figura 4, todos los párvulos y todas las párvulas avanzaron significativamente en las áreas estimuladas, fueron más significativos los casos 1 y 2 que alcanzaron sobre el $27 \%$ aproximadamente. Por lo tanto, las actividades de reconocimiento y análisis de los componentes fonéticos y silábicos, como las de simbolización del lenguaje y análisis de su estructura, que fueron realizadas con diferentes herramientas TIC, resultaron apropiadas para el desarrollar de aprendizajes en los niños y las niñas. 
doi: http://dx.doi.org/10.15359/ree.21-3.14

URL: http://www.una.ac.cr/educare

CORREO: educare@una.cr

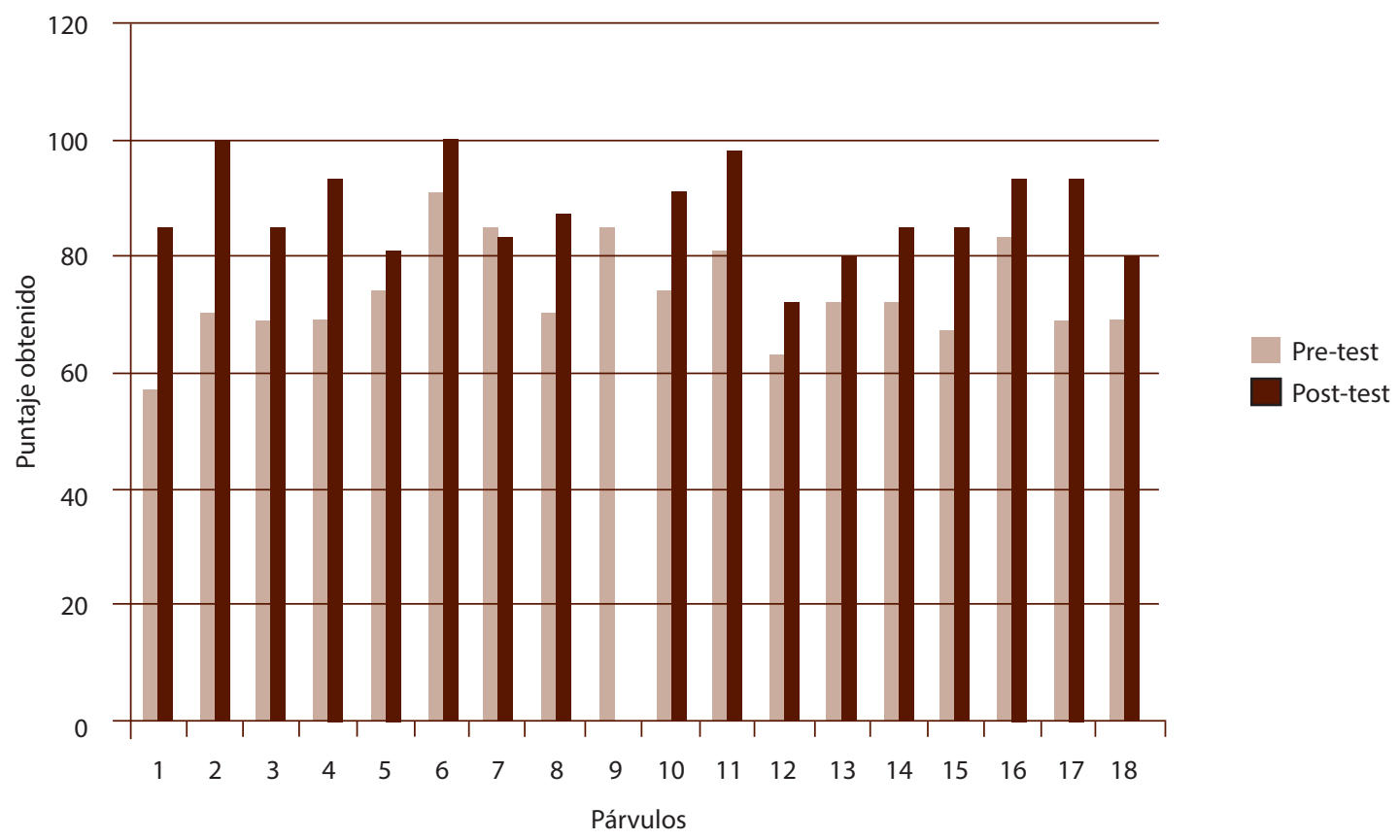

Figura 4: Resultado en porcentaje.

\section{Conclusiones}

Por medio de esta investigación se analizó el desarrollo de los aspectos fonológicos, semántico y sintáctico en los niños y las niñas. Gracias a los resultados obtenidos y el análisis cuantitativo, ha sido posible conocer los cambios que se produjeron al trabajar con diferentes herramientas TIC. Los resultados logrados permitieron explorar e intentar comprender cómo la niñez fue reflexionando sobre el lenguaje, separando la forma del significado (Medina, 1993).

Mediante las experiencias de aprendizajes que se realizaron con las herramientas TIC de realidad aumentada de imágenes en 3D de objetos, personas y animales, vídeos, páginas web, PowerPoint, y la pizarra digital interactiva, los niños y las niñas del nivel transición Il, lograron un mejor manejo de los aspectos semánticos, sintácticos y fonológicos.

Si bien, el análisis de los resultados proporciona información valiosa respecto a la utilización de las TIC en el aula, como lo señalan Arrieta y Delgado (2006), las animaciones y movimientos permitieron desarrollar mejor la habilidad para entender y pensar de las propias experiencias cognitivas y tomar conciencia de las situaciones en busca del descubrimiento del lenguaje. Sin embargo, se debe seguir investigando otras aplicaciones con estas herramientas, que permitan evidenciar el gran potencial didáctico que poseen. 


\section{Referencias}

Alegría, J. (1985). Por un enfoque psicolingüístico del aprendizaje de la lectura y sus dificultades. Revista Infancia y Aprendizaje, 29, 79-94. doi: https://doi.org/10.1080/02103702.1985.108 $\underline{22061}$

Alegría, J. (2006). Por un enfoque psicolingüístico del aprendizaje de la lectura y sus dificultades -20 años después-. Revista Infancia y Aprendizaje, 29(1), 93-111. Doi: http://dx.doi.org/10. 1080/02103702.1985.10822061

Andrade, B. (2012). Estudio del uso de la pizarra digital interactiva para desarrollar las competencias de los alumnos en el área de matemática en el nivel secundario (Tesis de pregrado). Universidad Tecnológiva Israel, Quito, Ecuador. Recuperado de http://190.11.245.244/ bitstream/47000/577/1/UISRAEL-EC-\%20SIS-\%20378.242-\%20254.pdf

Arrieta, X. y Delgado, M. (2006). Tecnologías de la información en la enseñanza de la física de educación básica. Revista Venezolana de Información, Tecnología y Conocimiento, 3(1), 6376. Recuperado de http://www.redalyc.org/articulo.oa?id=82330105

Hirumi, A., \& Grau, I. (1996). A review of computer-related state standards, textbooks, and journal articles. Journal of Computing in Teacher Education, 12(4), 6-17.

Azuma, R. T. (1997). A survey of augmented reality. Presence: Teleoperators and Virtual Environments, 6(4), 355-385. doi: https://doi.org/10.1162/pres.1997.6.4.355

Billinghurst, M. (2002). Augmented reality in education. Seattle WA: New Horizons for Learning. Recuperado de http://www.solomonalexis.com/downloads/ar edu.pdf

Bravo, L. (1990). Psicología de las dificultades del aprendizaje escolar. Santiago, Chile Editorial Universitaria.

Bravo, L., Villalón, M. y Orellana, E. (2002). La conciencia fonológica y la lectura inicial en niños que ingresan al primer año básico. Psykhé, 11(1), 175-182. Recuperado de http://www. psykhe.cl/index.php/psykhe/article/view/452/431

Briede, J. C., Leal, I. M., Mora, M. L. y Pleguezuelos C. S. (2015). Propuesta de modelo para el proceso de enseñanza-aprendizaje colaborativo de la observación en diseño, utilizando la pizarra digital interactiva (PDI). Formación Universitaria, 8(3), 15-26. doi: https://doi. org/10.4067/S0718-50062015000300003

Cooperstock, J. R. (2001). The classroom of the future: Enhancing education through augmented reality. Proceedings of $\mathrm{HCl}$ International, 688-692. Recuperado de http://srl.mcgill.ca/ publications/2001-HCl.pdf 
doi: http://dx.doi.org/10.15359/ree.21-3.14

URL: http://www.una.ac.cr/educare

CORREO: educare@una.cr

Enlaces Mineduc (2016). Proyecto Tablet para Educación Inicial. Orientaciones pedagógicas. Recuperado de file:///C:/Users/upla/Downloads/Proyecto Tablet Educ InicialOrientacionesPedagogicas.pdf

Díaz, C. y Álvarez-Salamanca, E. (2006). La importancia de potenciar tempranamente las competencias lingüísticas en la etapa inicial. Boletín de Investigación Educacional, 21(1), 115-137. Facultad de Educación. Pontificia Universidad Católica de Chile. Santiago.

Garassini, M. y Padrón, C. (2004). Experiencias de uso de las TIC en la educación preescolar en Venezuela. Anales de la Universidad Metropolitana, 4(1), 221-239. Recuperado de https:// dialnet.unirioja.es/servlet/revista?codigo $=14682$

Garton, A. F. (1994). Interacción social y desarrollo del lenguaje y la cognición. Barcelona: Paidós.

Hernández, R. Fernández, C. y Baptista, P. (2014). Metodología de la investigación (6a ed.). México: McGraw-Hill. Recupeado de http://upla.edu.pe/portal/wp-content/uploads/2017/01/ Hern\%C3\%A1ndez-R.-2014-Metodologia-de-la-Investigacion.pdf.pdf

Kerawalla, L., Luckin, R., Selijefot, S., \& Woolard, A. (2006). "Making it real": Exploring the potential of augmented reality for teaching primary school science. Virtual Reality, 10(3-4), 163-174. doi: https://doi.org/10.1007/s10055-006-0036-4

Klopfer, E., \& Squire, K. (2008). Environmental Detectives - the development of an augmented reality platform for environmental simulations. Educational Technology Research and Development, 56(2), 203-228. doi: https://doi.org/10.1007/s11423-007-9037-6

Marquès, P. y Casals, P. (2003). La pizarra digital en el aula de clase, una de las tres bases tecnológicas de la escuela del futuro. Revista Fuentes, 4, 1-9. Recuperado de https://ojs. publius.us.es/ojs/index.php/fuentes/article/view/2425/2294

Medina, M. (1993). Capacidades metalingüísticas: Un estudio de la detención y la explicación de la anomalía. Valle de Sartenejas: Ediciones de la Universidad Simón Bolivar.

Noda, A. (2009). Pizarra digital interactiva en aulas de matemáticas. Números. Revista de didáctica de las matemáticas, 72, 121-127. Recuperado de http://funes.uniandes.edu.co/3528/1/ Noda2009PizarraNumeros72.pdf

Orellana, E. (2000). La enseñanza del lenguaje escrito en un modelo interactivo. Pensamiento Educativo, 27(2), 15-34. Recuperado de http://pensamientoeducativo.uc.cl/index.php/ pel/article/view/175

Pinzás J. (2003). Metacongnición y lectura. Lima: Fondo editorial Pontificia PUCP. 
doi: http://dx.doi.org/10.15359/ree.21-3.14

URL: http://www.una.ac.cr/educare

Shelton, B., E., \& Hedley, N. R. (29 September, 2002). Using augmented reality for teaching earthsun relationships to undergraduate geography students. Paper presented at The First IEEE International Augmented Reality Toolkit Workshop, Darmstadt, Germany. Recuperado de http://ieeexplore.ieee.org/stamp/stamp.jsp?arnumber $=1106930$

Unesco. (2016). Tecnologías digitales al servicio de la calidad educativa. Una propuesta de cambio centrada en el aprendizaje para todos. Santiago: Autor. Recuperado de http:// unesdoc.unesco.org/images/0024/002451/245115S.pdf

Yanac, J. N. (2011). Desarrollo de un sistema web de realidad aumentada para apoyar la promoción del departamento de computación de la Universidad de Valparaíso (Tesis de posgrado). Universidad de Valparaíso, Chile. Recuperado de http://www.decom-uv.cl/rmunoz/tesis/ Juan\%20Yanac\%20Barreto/Doc\%20Tesis/Tesis\%20JYanac\%20-\%20VResumida.pdf 